\title{
White Matter Changes in Alzheimer's Disease Revealed by Diffusion Tensor Imaging with TBSS
}

\author{
Hongyan Chen', Kai Wang' ${ }^{2}$, Jingfan Yao ${ }^{2}$, Jianping Dai ${ }^{3}$, Jun $\mathrm{Ma}^{1}$, Shaowu $\mathrm{Li}^{3}$, Lin $\mathrm{Ai}^{4}$, \\ Qian Chen ${ }^{4}$, Xuzhu Chen" ${ }^{1}$, Yumei Zhang4* \\ ${ }^{1}$ Department of Radiology, Beijing Tiantan Hospital, Capital Medical University, Beijing, China \\ ${ }^{2}$ Capital Medical University, Beijing, China \\ ${ }^{3}$ Beijing Neurosurgical Insititute, Beijing, China \\ ${ }^{4}$ Department of Neurology, Beijing Tiantan Hospital, Capital Medical University, Beijing, China \\ Email: zhangyumei95@yahoo.com.cn
}

Received 8 January 2015; accepted 2 February 2015; published 5 February 2015

Copyright (C) 2015 by authors and Scientific Research Publishing Inc.

This work is licensed under the Creative Commons Attribution International License (CC BY).

http://creativecommons.org/licenses/by/4.0/

c) (i) Open Access

\begin{abstract}
Alzheimer's disease (AD) is a progressive, neurodegenerative disorder characterized by impairments in multiple cognitive domains and it is hard to diagnose in early stage because it's not easy to recognize and develop slowly. In this study, we try to evaluate the difference of white matter between AD and health volunteers using diffusion tensor imaging (DTI) and try to provide some evidence for diagnose AD in early stage. Twelve elderly Chinese patients with AD and twelve healthy volunteers were recruited and underwent DTI. The raw diffusion data were dealt with the toolkit of FSL image post-processing. Fractional anisotrogy (FA) data were then carried out by using tract-based spatial statistics (TBSS). The result showed that the FA of cingulum, hippocampus, corticospinal tract, and inferior fronto-occipital fasciculus significantly reduced in AD patients than that of volunteers. This indicated that the integrity of white matter tracts in these regions with AD was disturbed. On the other hand, the FA of other encephalic regions had no discrepancy compared with that of healthy volunteers. FA values were found reduced significantly in AD patients, especially in the posterior of the brain. These findings may provide image methods to diagnose patients with early stage of AD.
\end{abstract}

\section{Keywords}

Alzheimer Disease, Diffusion Tensor Imaging, Fractional Anisotropy, TBSS

\footnotetext{
${ }^{*}$ Corresponding author.
}

How to cite this paper: Chen, H.Y., Wang, K., Yao, J.F., Dai, J.P., Ma, J., Li, S.W., Ai, L., Chen, Q., Chen, X.Z. and Zhang, Y.M. (2015) White Matter Changes in Alzheimer's Disease Revealed by Diffusion Tensor Imaging with TBSS. World Journal of Neuroscience, 5, 58-65. http://dx.doi.org/10.4236/wjns.2015.51007 


\section{Introduction}

Alzheimer's disease (AD) is a primary neurodegenerative disorder characterized by progressive dementia at old age and early stage of old age [1]. It is a common neurodegenerative disease among the elderly. Most of AD cases progress slowly. However, with the disease development, cognitive function gradually declines, starting as impairment of memory, language, and visual-spatial ability at early stage that slowly develops into serious dysgnosia, catalogia and/or imitation speaking, etc. Neuro-psychological examinations provide certain sensitivity to recognize AD cognitive dysfunction at early stage, and at some level, it can help physicians to distinguish normal aging from $\mathrm{AD}[2]$ [3].

Most of the past AD imaging studies concentrated on the damage of gray matter (GM). However, studies of cadaver brains revealed that white matter (WM) changes were strongly associated with AD [4]-[7]. Study found that, the WM had significant pathological changes, including demyelinate and loss of axon, DNA breakage and lack of plasmalogen in AD patients at early stage [8].

Diffusion tensor imaging (DTI) is an imaging technology based on magnetic resonance diffusion weighted imaging, which can make quantitative analysis of anisotropy of water molecules in different directions, so as to observe the microstructure of tissues non-invasively. So DTI can provide information of fiber orientation, the injury of fiber, and membrane permeability which cannot be obtained from conventional MRI. DTI enables mapping of WM microstructure changes in development, aging and neurological disorders [9], and has therefore become a powerful technique in the study of neurodegenerative diseases in recent years. There are mainly two methods of DTI analysis; one is voxel-based analysis (VBA), and the other is TBSS. VBA is highly sensitive to registration errors and may produce false positives in affected regions [10]-[12]. TBSS can reduce the effects of local misregistrations by projecting all FA voxels onto the nearest location on a "skeleton” approximating WM tract center [9] [12] [13]. So TBSS has become a kind of popular software to study DTI, and has been used in some studies of various diseases, such as stroke [14], Parkinson [15], epilepsy [16], multiple sclerosis [17], traumatic brain injury [18], schizophrenia [19] and so on. Also some studies of cognitive disorders used TBSS, included mild cognitive impairment (MCI) and AD [9] [20].

At present, there is no single bio-indicator approved to diagnose AD; multiple examinations and comprehensive workup are required to make the diagnosis. There are only a few studies on DTI application in early stage AD. In our study, we applied DTI to analyze WM structural changes and its imaging characteristics of patients with AD at early stage. FSL data package was adopted to make image post-processing, and TBSS [13] based on fiber bundle skeleton was used to make statistical analysis of every voxel of FA data been tested.

\section{Subjects \& Methods}

\subsection{Subjects}

AD patients diagnosed and admitted by Department of Neurology of Tiantan Hospital during March, 2010 to March, 2011 and healthy volunteers with correspondent general conditions were recruited. AD group: all patients have met the following requirements. 1) Diagnostic criteria of the National Institute of Neurological and Communicative Disorders and Stroke and the Alzheimer's Disease and Related Disorders Association, NINCDSADRDA [21]; 2) Aged between 55 - 85 years old; 3) Scale test: determined by Mini Mental State Exam (MMSE), with a maximum score of 30 ; MMSE $\geq 27$ were considered as normal group; $\leq 17$ were considered as illiteracy group; $\leq 20$ were considered as elementary group; $\leq 22$ were considered as middle school group and would be diagnosed as having dementia; Hanchiski < 4; Hamilton anxiety and depression scale < 7; clinical dementia rating (CDR): 0 represented none, 1 represented mild, 2 represented moderate, and 3 represented severe; in Montreal Cognitive Assessment (MoCA), score $\geq 26$ were considered as normal; score $<26$ were considered as having cognitive dysfunction; in Activity of Daily Living (ADL), total score of 26 would be considered as completely normal; > 26 were considered to have decreased function of different degrees; total score of Alzheimer's Diseases assessment Scale-Cognitive section (ADAS-Cog) were 70; 4) Routine MRI showed no evidence of cerebral infarction, encephalic space occupying lesion, demyelination and cavity infarction; 5) No history of psychiatric disorders, congenital mental retardation or severe anxiety and depression, epilepsy, and alcohol or drug abuse; 6) No severe aphasis and could cooperate with examination; 7) No dementia caused by other conditions, including central nervous system injury, carcinoma, infection, metabolic disease, normal pressure hydrocephalus, folic acid and vitamin B12 deficiency, thyroid hypofunction, poisoning, etc.; 8) No severe heart, lung, 
liver and renal dysfunction or carcinoma.

A total of $12 \mathrm{AD}$ patients were enrolled, including 7 males and 5 females, with median age of (65.00 \pm 8.24$)$, median total school education length of $(8.17 \pm 3.86)$ years, and median MMSE score of $11.68 \pm 6.51$.

In the healthy control group, patients who received physical examination of the same period were recruited. The inclusion criteria were as follows: no neurological and systemic diseases; MRI test found no encephalic space occupying lesion, demyelination, infarction, and obvious encephalatrophy; no compliant about cognitive impairment and related clinical manifestations; scale test found no cognitive impairment; no psychiatric, personality and behavior disorders, etc.

All subjects had no metal implants in the body and MRI contraindications. Participation in this study was voluntary and all subjects have signed the informed consent form.

A total of 12 healthy volunteers were enrolled, including 7 males and 5 females, with median age of (64.33 \pm 8.56 years of old), median total school education length of (8.42 \pm 3.80$)$ years, and median MMSE score of $29.60 \pm 0.05$.

It was not necessary to do statistical test because the gender proportion of two groups were the same and sample size were relatively small. Independent sample t-test of general condition, age, and education level between two groups were performed, which found no statistical differences $(\mathrm{p}>0.05)$. Independent sample t-test of MMSE between two groups indicated significant differences $(0.001<\mathrm{p}<0.05$, see Table 1$)$. Independent sample t-test of other cognitive scales between two groups found significant differences $(0.001<\mathrm{p}<0.05$, see Table 2).

\subsection{Study Method}

\subsubsection{Cognitive Function Assessment}

This was carried out by professionals who have obtained certificates after completing neuro-psychological test training course by China Rehabilitation Center. All tests and data collection were performed after having obtained informed consent by patients and their family members. More than 60 patients participated in the test, among which, 36 patients quitted since they were unable to finish all tests due to illness and poor physical status. All enrolled patients strictly followed instructions and took examinations orderly. The examinations were carried out in a silent room from $5 \mathrm{pm}$ to $9 \mathrm{pm}$. Patients with poor general conditions needed a second examination

Table 1. Statistical analysis of general conditions of all subjects.

\begin{tabular}{ccccc}
\hline Group & Cases & Median age $(\mathrm{Y})$ & $\begin{array}{c}\text { Educational } \\
\text { background }(\mathrm{Y})\end{array}$ & MMSE (score) \\
\hline AD group & 12 & $65.00 \pm 8.24$ & $8.17 \pm 3.86$ & $11.68 \pm 6.51$ \\
NC group & 12 & $64.33 \pm 8.56$ & $8.42 \pm 3.80$ & $29.60 \pm 0.05$ \\
p value & 0.98 & 0.89 & $\mathrm{p}<0.001$ \\
p value & $\mathrm{p}>0.05$ & $\mathrm{p}>0.05$ & $\mathrm{p}<0.05$ \\
\hline
\end{tabular}

Note: AD group is Alzheimer's disease group; NC group is healthy control group.

Table 2. Statistical analysis of general conditions of other subjects.

\begin{tabular}{ccc}
\hline & AD & Control group \\
$\mathrm{N}$ & 12 & 12 \\
\hline MoCA (mean value \pm SD) & $17.11 \pm 2.63$ & $43.92 \pm 2.51$ \\
ADAS (mean value \pm SD) & $33.73 \pm 7.61$ & $53.82 \pm 11.91$ \\
ADL (mean value \pm SD) & $33.73 \pm 12.21$ & 0 \\
\hline CDR & 1 & 0.57 \\
\hline
\end{tabular}

Note: ADL, ADAS, and MoCA scores between the two groups had significant differences ( $<$ 0.0001). CDR: clinical dementia rating scale. MoCA: montreal cognitive assessment scale, ADL: daily life ability scale, ADAS: Alzheimer's diseases assessment scale-cognition. CDR: 1 represented mild; 2 represented moderate; 3 represented severe. 
to complete all items while most patients finished all items on first attempt.

\subsubsection{MRI}

DTI data was acquired on a Siemens 3.0 T Trio Tim MRI system with a 8 channels head coil using an echo planar imaging (EPI) sequence in 20 independent, non-collinear directions of a b-value $=1000 \mathrm{~s} / \mathrm{mm}^{2}$, and one additional image with no diffusion weighting $(\mathrm{b}=0)$. TR $=4900 \mathrm{~ms}$, TE $=93 \mathrm{~ms}$, field of view $(\mathrm{FOV})=230 \mathrm{~mm}$ $\times 230 \mathrm{~mm}$, imaging matrix $=64 \times 64$, number of slices $=36$, and slice thickness $=4 \mathrm{~mm}$ with no gap. Three acquisitions were averaged to increase the signal-to-noise ratio.

\subsubsection{Image Data Post-Processing and Statistical Methods}

All originaldata were converted into NIFTI format from DICOM format; deviation from movement and deformation were removed through registration techniques deviation; then FA Brain Mapping was calculated, and statistical analysis were made based on FA figure. FSL packet was used for offline DTI data post-processing. Some column's original diffusion weighted data were linear with Brigadier alignment, so as to minimize data deviation caused by head movement and eddy current effects. FA maps of the brain were calculated by BET algorithm to obtain location of the brain region. Then FA data of all subjects were aligned to a standardized Chinese brain space by using non-linear label. Based on the data of all subjects, the average FA maps can be calculated. In order to achieve reliable statistical results, it was necessary to create and extract an average FA skeleton. The skeleton corresponded to the fasciculus of white matter of the brain. Then each aligned FA data was projected onto this skeleton through the non-linear alignment. Finally, voxel-based FA statistical analysis method (TBSS) was used to find the differences between AD patients and normal control group within the space of the skeleton [13]. The threshold of FA value was 0.2 , ( $p<0.05$ indicated significant).

\section{Result}

FSL packet was used for DTI image data post-processing. TBSS statistical analysis results showed that: compared with the normal control group, patients belonging to the AD group had significant decrease in the FA values of the cingulate cortex (hippocampus), corticospinal tract, and inferior frontal white matter of the occipital fasciculus regions (Figures 1(A)-(C), respectively shown), which indicated the damage to the integrity of the

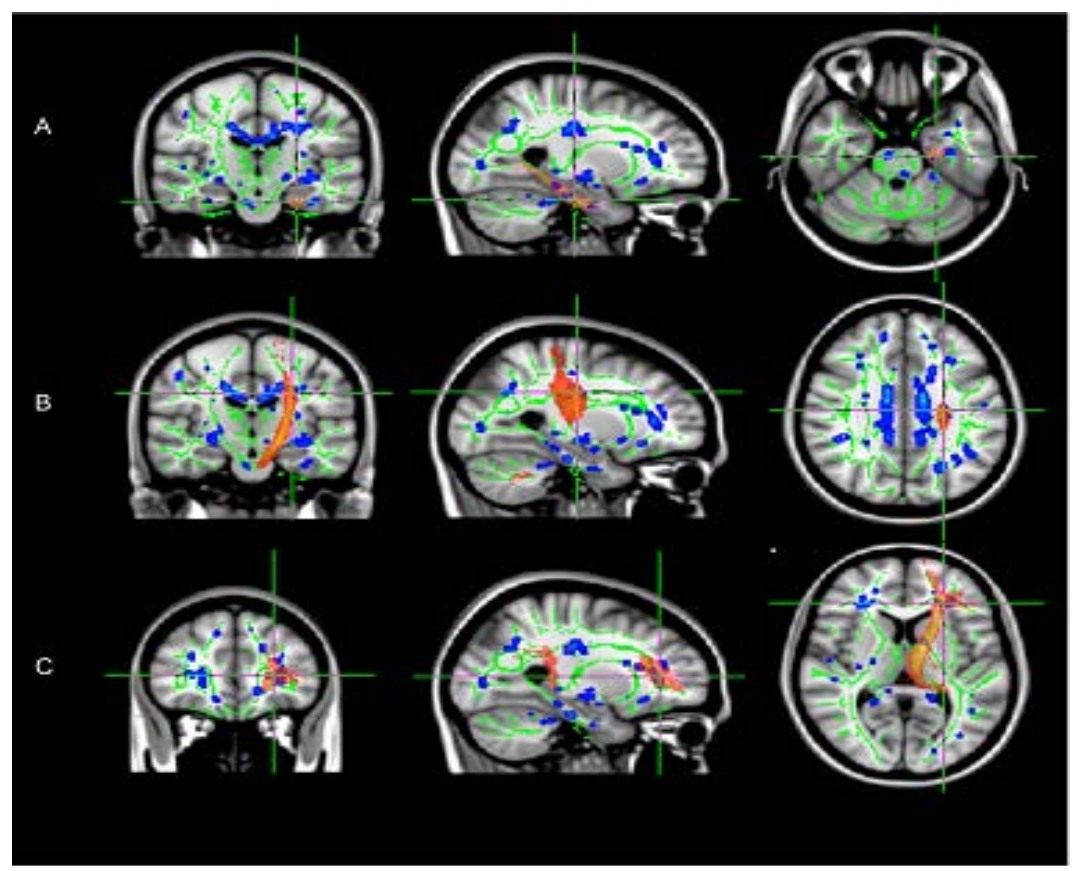

Figure 1. Green: average skeleton of white matter fiber bundles: blue: shows the areas where the FA value of AD patients was significantly less than that of the control patients; red: specific areas. (A) Cingulate cortex (hippocampus); (B) Corticospinal bundle of fiber bundles; (C) Inferior frontal occipital fasciculus. 
white matter fiber tracts in these regions. There was no significant statistical difference in FA values of other brain regions between $\mathrm{AD}$ and control group. These areas were generally located on the posterior part of the brain, indicating these $\mathrm{AD}$ patients suffered from regional damage of the white matter, and damage on the fiber integrity of the posterior brain was very prominent.

\section{Discussion}

DTI is a non-invasive imaging technology developed in recent years, which can be used for in vivo quantitative detection of the direction and extent of the diffusion of water molecules, thereby providing information on the capacity of the brain structure, direction and geometry, and also reflecting slight structure changes in white matter which would otherwise appear damage free on a traditional MRI. AD patients manifested selective regional damage of the white matter. In study by Medina et al. [22], compared with the control group, AD patients had significantly decreased FA value of temporal lobe subcortical white matter, corpus callosum posterior and posterior cingulate, indicating white matter damage of temporal lobe subcortical white matter, corpus callosum posterior and posterior cingulate, which was in consistency with neuropathological change in AD patients. Studies proved that white matter DTI changes occurred in early stage of $\mathrm{AD}$ and this can be used to reflect progression of AD [23] [24]. Study on AD patients at different stages by Nakata et al. [23] found, FA, MD and Easy Intelligence Scale scores were strongly associated, and were significantly correlated with the severity of Alzheimer's disease, which can be used to reflect the progression of Alzheimer's disease. Histopathological changes of the cingulate posterior white matter can be a useful biological marker to reflect Alzheimer's disease. A number of studies showed that the GA value, EADC value and FA values can be used as references for the imaging evaluation of patients with AD. These values of posterior cingulate fasciculus, superior longitudinal fasciculus, the posterior part of corpus callosum, and the WM of frontal, temporal, parietal lobes were correlated with neuropsychological test score, indicating that the changes in the integrity of white matter microstructure was one of the key factors leading to abnormal cognitive function in AD patients [24].

Autopsy and early DTI studies have demonstrated that AD pathology involve the gray matter and white matter [5] [25]. Both normal aging and AD can cause atrophy, demyelination and degeneration of the white matter [26]. Some studies claimed that white matter abnormalities caused by aging were located mainly in the anterior part of the brain [27], while white matter abnormalities caused by AD, like gray matter, were located mainly in the posterior [27]. However, which area of white matter is involved at early stage of AD has not yet been determined. Therefore, in this study, patients with early stage AD were enrolled. This study found that, compared with normal control group, AD group's FA values of the cingulate gyrus (hippocampus), corticospinal tract, and inferior frontal occipital fasciculus white matter was decreased significantly, in particular to posterior brain regions. FA values reflect white matter integrity. Our study results showed that, in the early stages of $\mathrm{AD}$, the cingulate gyrus (hippocampus), the corticospinal tract, inferior frontal occipital fasciculus white matter integrity has been affected, and this abnormality was associated with cognitive impairment. Some DTI studies also supported the results of this study, such as showing significant FA values decrease of the posterior cingulate and parahippocampal gyrus white matter [22]-[24] [28] [29], which indicated that the white matter micro-structure of the limbic system of the AD patients were also affected. The limbic system includes the hippo-campus, parahippocampal gyrus, entorhinal, dentate gyrus, cingulate gyrus, mammillary body and amygdala gray matter structures. In neuropsychological level, the limbic system is mainly associated with memory and emotion, and memory impairment is the earliest and most obvious neuropsychological symptom of AD. The performance of the group of patients with $\mathrm{AD}$ also showed related cognitive decline, which confirmed the reliability of the findings. Damoiseaux et al. [30] found that FA values of the inferior frontal occipital fasciculus declined. Inferior frontal occipital fasciculus connects the lower part of the frontal lobe and lower posterior occipital lobe, and helps to form the temporal stem, the hook beam and inferior frontal occipital fasciculus as well as optic radiation getting through the front and posterior of the temporal stem. In addition, the inferior frontal occipital fasciculus joins the left fusiform gyrus to form the pathway of ventral semantics. Damage to the path may lead to the dysfunctions of the visual word form [31]. During cognitive testing, the study also found that some patients have reading disability, supporting the above point. In addition, the study also found that there is damage to the corticospinal tract, which is different from the results of some studies [32]. It is speculated to be related to the emergence of early motor dysfunction in AD patients.

Above mentioned results suggested that, in patients with early AD, white matter microstructure had selective 
damage and the integrity of white matter pathways was destroyed. The selective damage may be related to the distribution of the cortex and white matter pathways senile plaques and neuronal neurofibrillary tangles [9]. In the early stages of dementia, white matter integrity of cingulate gyrus (hippocampus), the corticospinal tract, and inferior frontal occipital fasciculus had been affected, which can explain why memory dysfunction and dyslexia appear in the early stage of AD.

Clinical neuropsychological assessment scale is a comprehensive assessment that reflects a variety of brain functions at the same time and not designed for assessing function of focal brain regions, and one brain function often involves multiple brain regions at the same time, so any focal lesions of the brain may lead to impairment of a certain function. Therefore, many factors might have affected on this study, which need to use specialized cognitive scale. Secondly, the DTI data we collected were analyzed by using the cellulose-based spatial statistical analysis method. The inherent shortcomings of the method in the registration of statistical structure projected onto the skeleton, so the difference is confined to the skeleton, which is corresponding to white matter fiber prime and other regional differences are ignored. In addition, number of cases and control sample size in this study were relatively small.

\section{Conclusion}

In short, increase in the number of patients and controls, long-term follow-up, a more professional scale, and optimized imaging methods and data analysis methods, will be more conducive to the study of the white matter in AD patients.

\section{References}

[1] Dubois, B., Feldman, H.H., Jacova, C., et al. (2007) Research Criteria for the Diagnosis of Alzheimer's Disease: Revising the NINCDS-ADRDA Criteria. Lancet Neurology, 6, 734-746. http://dx.doi.org/10.1016/S1474-4422(07)70178-3

[2] Alzheimer's Association (2011) Alzheimer’s Disease Facts and Figures. Alzheimer's \& Dementia, 7, $208-244$. http://dx.doi.org/10.1016/j.jalz.2011.02.004

[3] Klöppel, S., Stonnington, C.M., Chu, C., et al. (2008) Automatic Classification of MR Scans in Alzheimer's Disease. Brain, 131, 681-689. http://dx.doi.org/10.1093/brain/awm319

[4] Teipel, S.J., Stahl, R., Dietrich, O., et al. (2007) Multivariate Network Analysis of Fiber Tract Integrity in Alzheimer's Disease. NeuroImage, 34, 985-995. http://dx.doi.org/10.1016/j.neuroimage.2006.07.047

[5] Brun, A. and Englund, E. (1986) A White Matter Disorder in Dementia of the Alzheimer Type: A Patho-Anatomical Study. Annals of Neurology, 19, 253-262. http://dx.doi.org/10.1002/ana.410190306

[6] Salat, D.H., Tuch, D.S., van der Kouwe, A.J., et al. (2010) White Matter Pathology Isolates the Hippocampal Formation in Alzheimer's Disease. Neurobiol Aging, 31, 244-256. http://dx.doi.org/10.1016/j.neurobiolaging.2008.03.013

[7] Francesca, B., Marina, S., Maria, G.P., Monia, C., Ludovica, G., Ivana, M., Federica, P., Elena, C., Raffaello, N. and Mario, C. (2013) Neuroinflammation and Brain Functional Disconnection in Alzheimer's Disease. Frontiers in Aging Neuroscience, 5, 81.

[8] Villain, N., Desgranges, B., Viader, F., et al. (2008) Relationships between Hippocampal Atrophy, White Matter Disruption, and Gray Matter Hypometabolism in Alzheimer's Disease. Neuroscience, 28, 6174-6181. http://dx.doi.org/10.1523/JNEUROSCI.1392-08.2008

[9] Julio, A.C. and Perter, J.N. (2014) Diffusio Tensor Imaging in Alzherimer's Disease: Insights into the Limbic-Diencephalic Network and Methodological Considerations. Frontiers in Aging Neuroscience.

[10] Ashburner, J. and Friston, K.J. (2000) Voxel-Based Morphometry-The Methods. NeuroImage, 11, 805-821. http://dx.doi.org/10.1006/nimg.2000.0582

[11] Bookstein, F.L. (2001) "Voxel-Based Morphometry” Should Not Be Used with Imperfectly Registered Images. NeuroImage, 14, 1454-1462. http://dx.doi.org/10.1006/nimg.2001.0770

[12] Schwarz, C.G., Reid, R.I., Gunter, J.L., Senjem, M.L., Przybelski, S.A., Zuk, S.M., Whitwell, J.L., Vemuri, P., Josephs, K.A., Kantarci, K., Thompson, P.M., Petersen, R.C. and Jack Jr., C.R., Alzheimer's Disease Neuroimaging Initiative (2014) Improved DTI Registration Allows Voxel-Based Analysis That Outperforms Tract-Based Spatial Statistics. NeuroImage, 94, 65-78. http://dx.doi.org/10.1016/j.neuroimage.2014.03.026

[13] Smith, S.M., Jenkinson, M., Johansen-Berg, H., Rueckert, D., Nichols, T.E., Mackay, C.E., et al. (2006) Tract-Based Spatial Statistics: Voxelwise Analysis of Multi-Subject Diffusion Data. NeuroImage, 31, 1487-1505. 
http://dx.doi.org/10.1016/j.neuroimage.2006.02.024

[14] Dacosta-Aguayo, R., Graña, M., Fernández-Andújar, M., López-Cancio, E., Cáceres, C., Bargalló, N., Barrios, M., Clemente, I., Monserrat, P.T., Sas, M.A., Dávalos, A., Auer, T. and Mataró, M. (2014) Structural Integrity of the Contralesional Hemisphere Predicts Cognitive Impairment in Ischemic Stroke at Three Months. PLoS ONE, 9, e86119. http://dx.doi.org/10.1371/journal.pone.0086119

[15] Perea, R.D., Rada, R.C., Wilson, J., Vidoni, E.D., Morris, J.K., Lyons, K.E., Pahwa, R., Burns, J.M. and Honea, R.A. (2013) A Comparative White Matter Study with Parkinson's Disease, Parkinson's Disease with Dementia and Alzheimer's Disease. Journal of Alzheimer's Disease Parkinsonism, 3, 123.

[16] Scanlon, C., Mueller, S.G., Cheong, I., Hartig, M., Weiner, M.W. and Laxer, K.D. (2013) Grey and White Matter Abnormalities in Temporal Lobe Epilepsy with and without Mesial Temporal Sclerosis. Journal of Neurology, 260, 2320 2329. http://dx.doi.org/10.1007/s00415-013-6974-3

[17] Shen, Y., Bai, L., Gao, Y., Cui, F., Tan, Z., Tao, Y., Sun, C. and Zhou, L. (2014) Depressive Symptoms in Multiple Sclerosis from an in Vivo Study with TBSS. BioMed Research International, 2014, 1-8. http://dx.doi.org/10.1155/2014/148465

[18] Zhu, Y., Li, Z., Bai, L., Tao, Y., Sun, C., Li, M., Zheng, L., Zhu, B., Yao, J., Zhou, H. and Zhang, M. (2014) Loss of Microstructural Integrity in the Limbic-Subcortical Networks for Acute Symptomatic Traumatic Brain Injury. BioMed Research International, 2014, 1-7. http://dx.doi.org/10.1155/2014/548392

[19] Roalf, D.R., Ruparel, K., Verma, R., Elliott, M.A., Gur, R.E. and Gur, R.C. (2013) White Matter Organization and Neurocognitive Performance Variability in Schizophrenia. Schizophrenia Research, 143, 172-178. http://dx.doi.org/10.1016/j.schres.2012.10.014

[20] Liu, J., Yin, C., Xia, S., Jia, L., Guo, Y., Zhao, Z., Li, X., Han, Y. and Jia, J. (2013) White Matter Changes in Patients with Amnestic Mild Cognitive Impairment Detected by Diffusion Tensor Imaging. PLoS ONE, 8, e59440. http://dx.doi.org/10.1371/journal.pone.0059440

[21] Medin, D., de Toledo-Morrell, L., Urresta, F., Gabrieli, J.D.E., Moseley, M., Fleischman, D., et al. (2006) White Matter Changes in Mild Cognitive Impairment and AD: A Diffusion Tensor Imaging Study. Neurobiology of Aging, 27, 663-672. http://dx.doi.org/10.1016/j.neurobiolaging.2005.03.026

[22] Chua, T.C., Wen, W., Slavin, M.J. and Sachdev, P.S. (2008) Diffusion Tensor Imaging in Mild Cognitive Impairment and Alzheimer's Disease: A Review. Current Opinion in Neurology, 21, 83-92. http://dx.doi.org/10.1097/WCO.0b013e3282f4594b

[23] Rose, S.E., Janke, A.L. and Chalk, J.B. (2008) Gray and White Matter Changes in Alzheimer's Disease: A Diffusion Tensor Imaging Study. Journal of Magnetic Resonance Imaging, 27, 20-26. http://dx.doi.org/10.1002/jmri.21231

[24] Nakata, Y., Sato, N., Nemoto, K., Abe, O., Shikakura, S., Arima, K., et al. (2009) Diffusion Abnormality in the Posterior Cingulum and Hippocampal Volume: Correlation with Disease Progression in Alzheimer's Disease. Magnetic Resonance Imaging, 27, 347-354. http://dx.doi.org/10.1016/j.mri.2008.07.013

[25] Ashburner, J. and Friston, K.J. (2000) Voxel-Based Morphometry the Methods. NeuroImage, 11, 805-821. http://dx.doi.org/10.1006/nimg.2000.0582

[26] Hugenschmidt, C.E., Peiffer, A.M., Kraft, R.A., Casanova, R., Deibler, A.R., Burdette, J.H., et al. (2007) Relating Imaging Indices of White Matter Integrity and Volume in Healthy Older Adults. Cerebral Cortex, 18, 433-442. http://dx.doi.org/10.1093/cercor/bhm080

[27] Damoiseaux, J.S., Smith, S.M., Witter, M.P., Sanz-Arigita, E.J., Barkhof, F., Scheltens, P., et al. (2009) White Matter Tract Integrity in Aging and Alzheimer's Disease. Human Brain Mapping, 30, 1051-1059. http://dx.doi.org/10.1002/hbm.20563

[28] Zhang, Y., Sehuff, N., Jahng, G.H., Bayne, W., Mori, S., Schad, L., et al. (2007) Diffusion Tensor Imaging of Cingulum Fibers in Mild Cognitive Impairment and Alzhelmer Disease. Neurology, 68, 13-19. http://dx.doi.org/10.1212/01.wnl.0000250326.77323.01

[29] Rose, S.E., McMahon, K.L., Janke, A.L., O’Dowd, B., de Zubicaray, G., Strudwick, M.W. and Chalk, J.B. (2006) Diffusion Indices on Magnetic Resonance Imaging and Neuropsychological Performance in Amnestic Mild Cognitive Impairment. Journal of Neurology, Neurosurgery \& Psychiatry, 77, 1122-1128. http://dx.doi.org/10.1136/jnnp.2005.074336

[30] Duffau, H., Gatignol, P., Mandonnet, E., Peruzzi, P., Tzourio-Mazoyer, N. and Capelle, L. (2005) New Insights into the Anatomo-Functional Connectivity of the Semantic System: A Study Using Cortico-Subcortical Electrostimulations. Brain, 128, 797-810. http://dx.doi.org/10.1093/brain/awh423

[31] Choi, S.J., Lim, K.O., Monteiro, L. and Reisberg, B. (2005) Diffusion Tensor Imaging of Frontal White Matter Microstructure in Early Alzheimer's Disease: A Preliminary Study. Journal of Geriatric Psychiatry and Neurology, 18, 12 19. http://dx.doi.org/10.1177/0891988704271763 
[32] Teipel, S., Ehlers, I., Erbe, A., Holzmann, C., Lau, E., Hauenstein, K. and Berger, C. (2014) Structural Connectivity Changes Underlying Altered Working Memory Networks in Mild Cognitive Impairment: A Three-Way Image Fusion Analysis. Journal of NeuroImaging. http://dx.doi.org/10.1111/jon.12178 
Scientific Research Publishing (SCIRP) is one of the largest Open Access journal publishers. It is currently publishing more than 200 open access, online, peer-reviewed journals covering a wide range of academic disciplines. SCIRP serves the worldwide academic communities and contributes to the progress and application of science with its publication.

Other selected journals from SCIRP are listed as below. Submit your manuscript to us via either submit@scirp.org or Online Submission Portal.
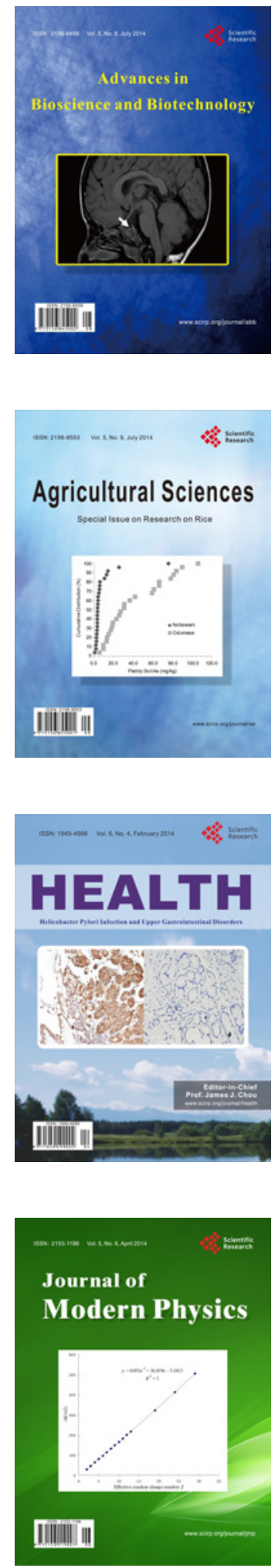
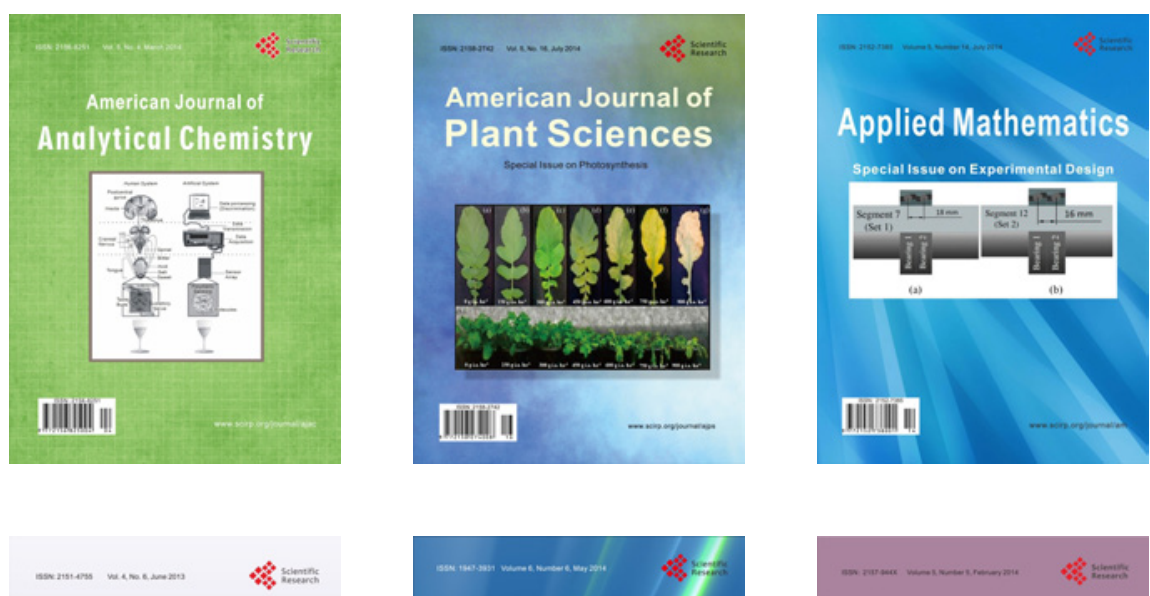

Creative Education
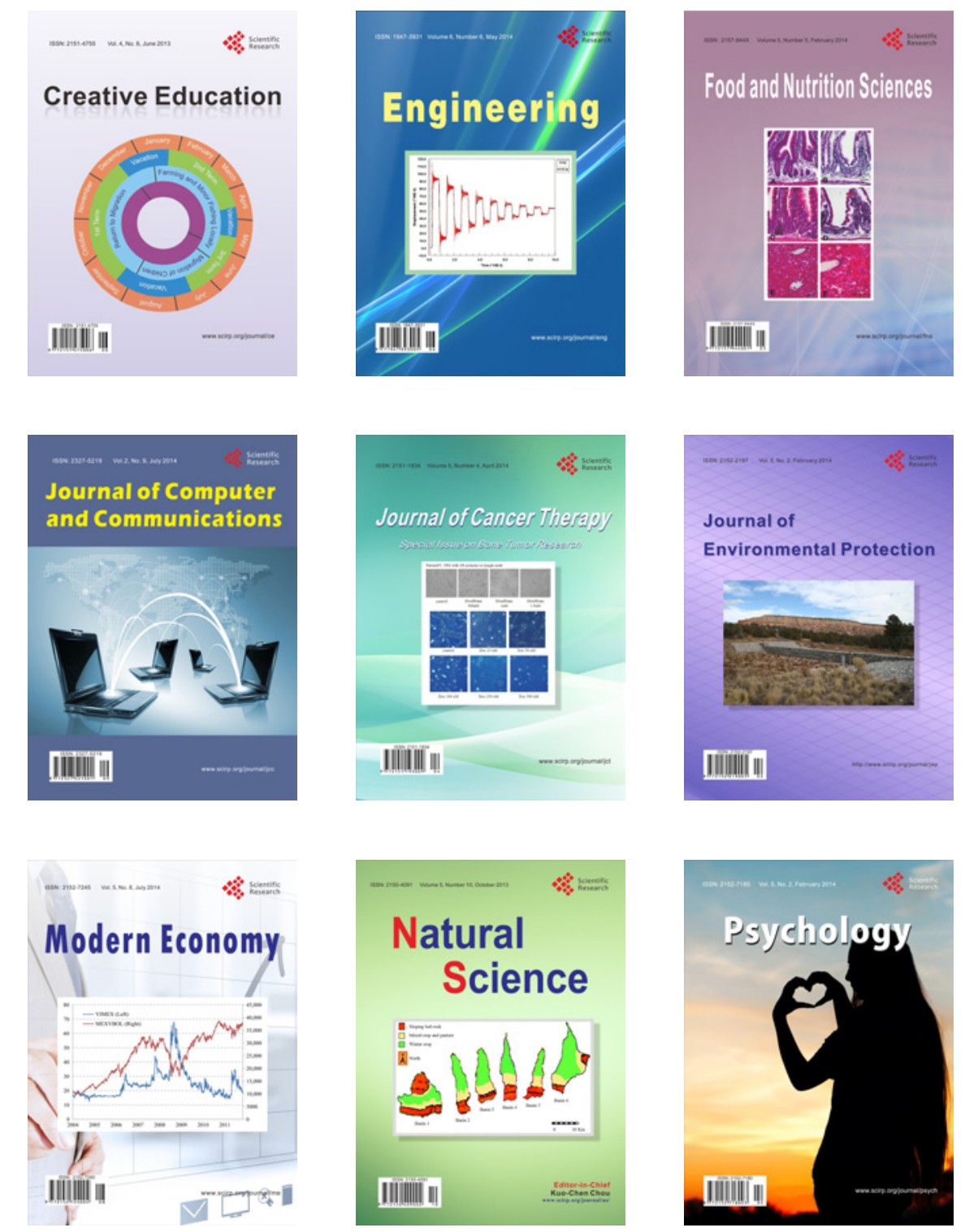